\title{
Influence of the Laser Beam Shape on Laser-Induced Periodic Surface Structure Formation Assisted by Surface Plasmon Polaritons
}

\author{
J. Oltmanns ${ }^{1, *, \#, ~ P . N . ~ T e r e k h i n ~}{ }^{2, \#}$, F. Kleinwort ${ }^{1,}$ A. Blumenstein ${ }^{1}$, D.S. Ivanov ${ }^{2,3,4}$, M.E. Garcia ${ }^{4}$, B. Rethfeld ${ }^{2}$, \\ J. Ihlemann ${ }^{1}$, and P. Simon ${ }^{1}$ \\ ${ }^{1}$ Institut für Nanophotonik Göttingen e.V., Hans-Adolf-Krebs-Weg 1, 37077 Göttingen, Germany \\ ${ }^{2}$ Department of Physics and Research Center OPTIMAS, Technische Universität Kaiserslautern, \\ Erwin-Schrödinger-Straße 46, 67663 Kaiserslautern, Germany \\ ${ }^{3}$ Quantum Electronics Division, Lebedev Physical Institute, 119991 Moscow, Russia \\ ${ }^{4}$ Theoretical Physics Department, University of Kassel, 34132 Kassel, Germany \\ ${ }^{*}$ Contributed Equally \\ ${ }^{*}$ Corresponding author's e-mail: jens.oltmanns@ifnano.de
}

\begin{abstract}
The ability to control the properties of laser-induced periodic surface structures (LIPSS) is highly demanded for advanced technologies. We have studied the surface plasmon polaritons assisted creation of LIPSS in the vicinity of a predesigned gold step edge applying single-pulse femtosecond laser irradiation. We show that the heights of LIPSS can be manipulated by adjusting the laser beam shape. Our results pave the way towards controllable single-pulse laser nanostructuring.

DOI: 10.2961/jlmn.2021.03.2009
\end{abstract}

Keywords: LIPSS, ripples, nanostructuring, surface modification, femtosecond lasers

\section{Introduction}

Irradiating a material with a laser beam may lead to the formation of periodic structures on the sample surface. These structures are well known as laser-induced periodic surface structures (LIPSS) and were first observed in 1965 by Birnbaum [1]. Since then these structures have been observed on many different materials using lasers of various wavelengths, pulse durations and applying different fluences and number of pulses [2,3]. LIPSS are used for the functionalization of surfaces in many fields, including industrial, medical, biological and optical applications [2-4].

There are different theories about the processes involved in the formation of LIPSS [3]. One promising theory to explain the creation of LIPSS on metal surfaces is via the coupling of surface plasmon polaritons (SPP), i.e. electromagnetic waves excited by the laser beam and propagating along the dielectric/metal interface. The SPP may interfere with the laser pulse and result in a periodically modulated energy deposition onto the sample [2,3,5-7].

SPP are well studied at low pulse energies and are used for the investigation of SPP waveguides [8], nano-antennas $[9,10]$ as well as other applications [11]. To excite the SPP, there has to be a defect on the metal surface. Quite often, LIPSS are created by applying multiple pulses onto the sample surface $[3,12,13]$, then the first few pulses create defects on the sample while the later ones excite the SPP. As the morphology of the surface changes with each pulse, it is hard to understand in detail the fundamental processes involved in the creation of LIPSS.

Only a few publications examine the creation of LIPSS after single pulse irradiation [14-20]. It has been pointed out in Ref. [3] that in the case of high fluences already a prepulse may be strong enough to modify the sample surface and create some defects. These defects may drive the creation of SPP and subsequently LIPSS at the main-pulse.
To model the energy absorption of the laser pulse by the metallic sample, often the well-known two-temperature model (TTM) [21,22] is applied. The TTM also allows for describing the evolution of the energy transfer by tracking the temperature dynamics of the electron subsystem and that of the lattice. In this model, the electron subsystem gains energy through the source term, which describes the energy input per time and volume into the sample.

The TTM model with an additional equation for the carrier density was applied in Ref. [23] to investigate LIPSS formation on silicon. The authors found that the increase of the electron-hole free carriers' density in the conduction band of silicon due to one- or multi-photon absorption process can reach a level sufficient for the excitation of SPP waves. In other works, e.g. [19,24-26], an arbitrary period in the modulation of the source term was assumed. With the same assumption Refs. $[27,28]$ studied the creation of LIPSS on chromium in the framework of a combined approach of molecular dynamic simulation and two-temperature description (MD-TTM) [29,30]. These models demonstrate the process of energy deposition in the material, but leave out the SPP periodicity and decay length, which, when included, would lead to different morphologies that are better comparable to experimental results.

In this publication, we experimentally investigate the process of the single-pulse LIPSS formation on a gold sample in the vicinity of a pre-existing step edge. We also demonstrate the possibility of manipulation of the LIPSS modulation depth via the laser beam shaping. 


\section{Theory}

If a laser pulse hits a smooth surface, SPP cannot be excited as there is a mismatch in linear momentum between the two waves (SPP and light). However, if the surface contains a defect, the geometry may provide the missing linear momentum. That makes it possible to launch SPP waves using a step edge [31,32] or a rough surface [33]. Additionally, it is possible to excite SPP waves on a smooth surface using a prism setup [33,11]. A sketch of the process of SPP excitation on a step edge is shown in Fig. 1.

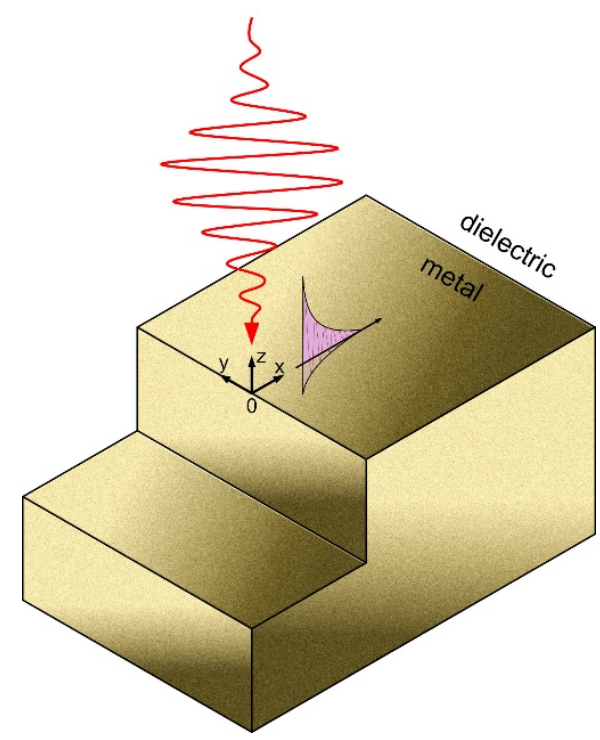

Fig. 1 Scheme of the step edge setup for SPP excitation, which may lead to LIPSS formation on the surface. A laser pulse with p-polarization hits the step edge on the sample surface. Afterwards the SPP is excited and travels along the surface perpendicular to the alignment of the step edge.

Another requirement for the excitation of SPP by laser irradiation at air/metal interface is related to the complex dielectric function (CDF) $\varepsilon_{\mathrm{m}}$ of the metal. The metal must fulfil at the given wavelength of the laser $\lambda_{\text {Las }}$ the requirement $\operatorname{Re}\left(\varepsilon_{\mathrm{m}}\left(\lambda_{\text {Las }}\right)\right)<0$. The SPP will be excited at the step edge by the p-polarized component of the laser pulse and move perpendicular to the step edge. The SPP wave may then interfere with the still incoming laser pulse and form an interference pattern.

The dispersion relation of the SPP wave depends on the $\mathrm{CDF}$ of the metal and the dielectric on top as well as the laser wavelength. It has the form

$$
k_{S P P}=\frac{2 \pi}{\lambda_{\text {Las }}} \sqrt{\frac{\varepsilon_{m} \varepsilon_{d}}{\varepsilon_{m}+\varepsilon_{d}}} .
$$

If the dielectric is air, the dispersion relation simplifies to

$$
k_{S P P}=\frac{2 \pi}{\lambda_{\text {Las }}} \sqrt{\frac{\varepsilon_{m}}{\varepsilon_{m}+1}} .
$$

The wavelength of the SPP $\lambda_{\text {Spp }}$ also depends on the CDF and the laser wavelength. It can be calculated via

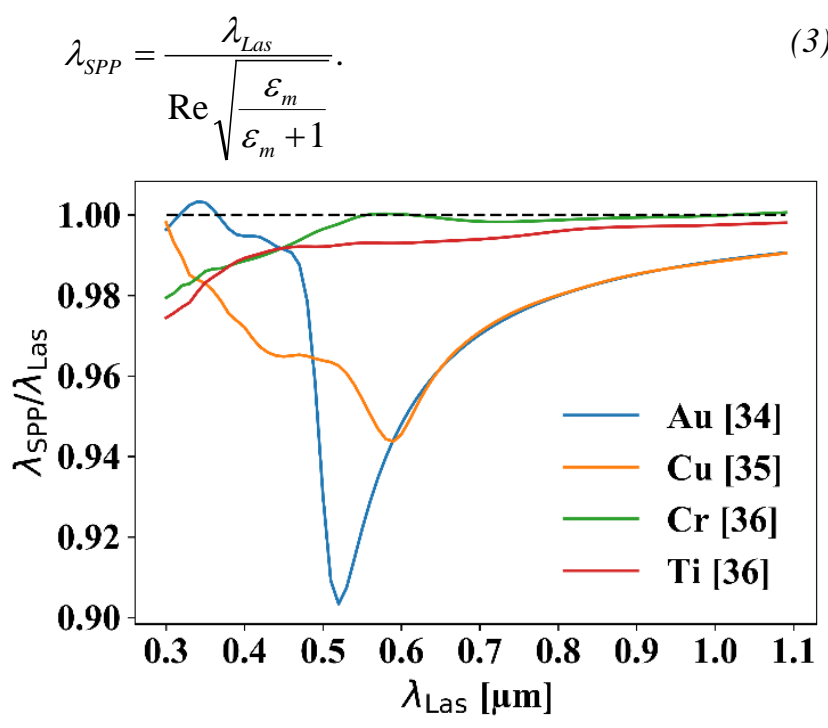

Fig. 2 The calculated wavelength of the SPP using Eq. (3) normalized by the laser wavelength for Gold (Au) [34], Copper (Cu) [35], Chromium (Cr) [36] and Titanium (Ti) [36].

It can be seen in Fig. 2 that generally the SPP wavelength $\lambda_{\text {SpP }}$ is lower than the laser wavelength for the presented metals in the given range of laser wavelengths. The SPP wavelength $\lambda_{\text {SPP }}$ tends to the laser wavelength $\lambda_{\text {Las }}$ with increasing $\lambda_{\text {Las. }}$ Air / Au and air / Cu systems show certain dips, which are related to the d-band excitation thresholds of $\mathrm{Au}$ and $\mathrm{Cu}$, correspondingly. In contrast, the SPP wavelengths $\lambda_{\text {SPP }}$ for $\mathrm{Cr}$ and $\mathrm{Ti}$ are very close to the laser wavelength $\lambda_{\text {Las }}$ in the whole investigated range of wavelengths.

\section{Experimental Setup}

The used sample consists of a gold layer produced via deposition by evaporation with a thickness of about $350 \mathrm{~nm}$ on a thin chromium layer on a glass substrate. The chromium layer is needed to increase the adhesion of the gold layer to the substrate. The surface roughness has been measured using an atomic force microscope (AFM), it gave a quadratic surface roughness of about $1 \mathrm{~nm}$. To create the step edge on the surface, a razor blade has been used. The blade was pressed onto the sample surface and the sample moved underneath it using a motorized stage. This led to a scratch which is about $40 \mu \mathrm{m}$ broad and has uplifted walls on the sides. The walls have a height of $100-200 \mathrm{~nm}$. One of the walls was used as step edge for the experiments. All experiments were done using the right edge of the scratch displayed in Fig. 3. The appearance of the edge varies slightly along the scratch, but no changes in the LIPSS could be traced back to variations along the edge. The laser beam was always centered on the selected wall. An AFM measurement of the produced step edge with the corresponding height profile can be seen in Fig. 3. As the distance between the two edges of the scratched groove is about $20 \mu \mathrm{m}$, it may happen that the outer part of the beam hits the other edge as well. Due to the fast decay of the SPP we do not expect an influence of this effect on the final structures, especially as the beam energy at that location is rather low. 


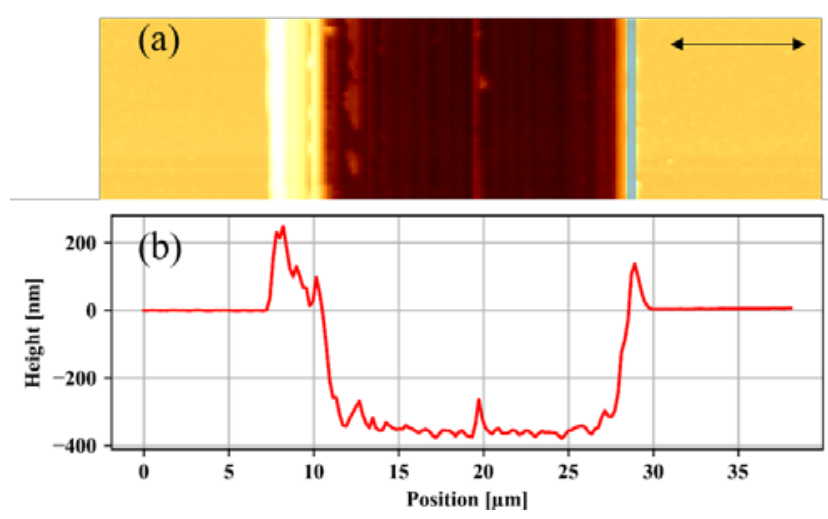

Fig. 3 (a) AFM measurement of the scratch created by a razor blade pressed against the sample surface. The fade blue line indicates the position of the center of the beam for later irradiation and the black arrow shows the polarization of the beam. (b) Height profile of the scratch with walls forming on the sides by the pushed out gold from the scratch. The wall height is between 100-200 nm.

The setup of the experiment consists of a light source (Light Conversion Pharos $\mathrm{PH}-1-20 \mathrm{~W}$ ) with a fundamental wavelength of $1030 \mathrm{~nm}$. In the experiments, the third harmonic radiation with a wavelength of $343 \mathrm{~nm}$, a spectral broadness of about $1 \mathrm{~nm}$ and a pulse length of about $200 \mathrm{fs}$ was used.

The polarization of the laser is controlled by a $\lambda / 2$ waveplate and a Glan-Taylor polarizer and was set perpendicular to the step edge. Afterwards, the beam passes a Gauss-toTop-hat (G2T) beam shaper (FBS2-40-343, TOPAG Lasertechnik $\mathrm{GmbH}$ ). The beam is then focused on the sample using a lens with a focal length of $f=250 \mathrm{~mm}$. The size of the resulting nearly homogeneous focus $(w)$ can be estimated by $w=\frac{2 \lambda f}{d}$ with $d$ being the initial beam diameter. In this case for a beam with $d=4 \mathrm{~mm}\left(1 / \mathrm{e}^{2}\right)$, the focus is about $40 \mu \mathrm{m}$, measured where the intensity decreases to $1 / \mathrm{e}^{2}$. The irradiations took place under normal incidence with a p-polarized beam. A sketch of the setup is displayed in Fig. 4.

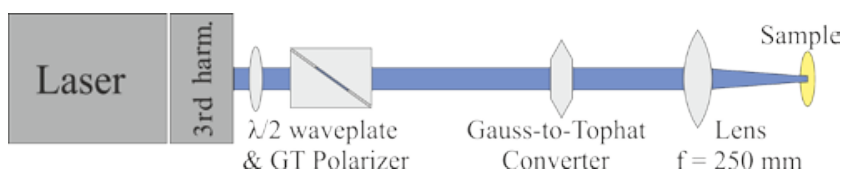

Fig. 4 The used experimental setup consisting of a femtosecond laser source, a $\lambda / 2$ wave plate and a Glan-Taylor polarizer to control the polarization of the beam and a G2T with a focusing lens to create specific beam profiles on the sample surface.

Moving the G2T sideways allows for modifying the fluence distribution so that a slope is obtained with a fluence peak at the right side. The experiments were done using both a homogeneously shaped beam profile and a profile with a peak fluence shifted to the right side. Both profiles have a size of about $40 \mu \mathrm{m}\left(1 / \mathrm{e}^{2}\right)$ and are shown in Fig. 5 . The height measurements of the sample are done using an AFM (XE-150, Park). The measured area has a size of $8 \mu \mathrm{m}$ in both $\mathrm{x}$ and $\mathrm{y}$ direction with the step edge on the very left side of the measurement.

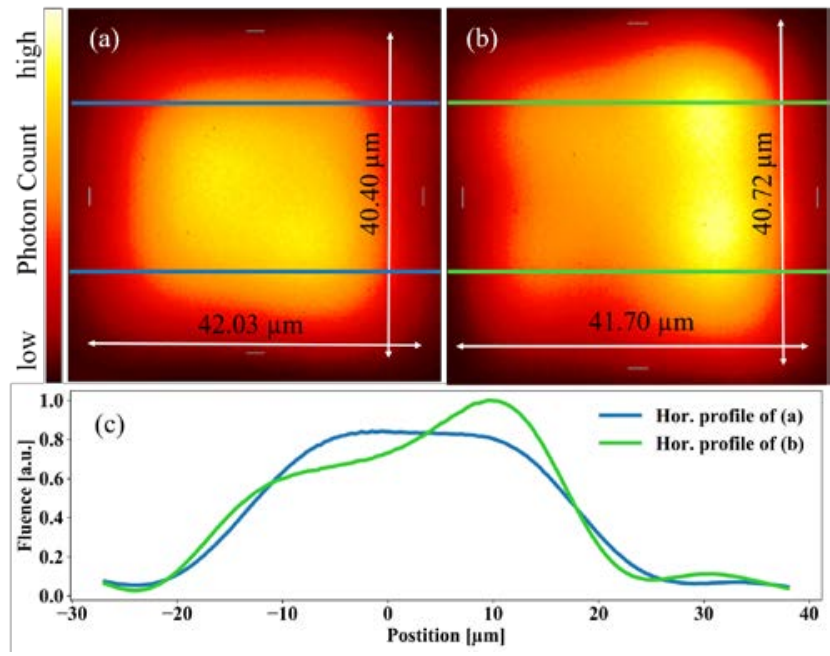

Fig. 5 Beam profiles used for irradiations. (a) A homogeneous beam profile and (b) a profile with a fluence slope. (c) shows the fluence distribution along a horizontal line for both beam profiles, averaged over 25 rows in the regions between the blue and green lines, respectively. The pictures are taken by placing a Lumenera Lw165 camera with a 20x magnification objective at the position of the sample. The recorded profile was analyzed using the MrBeam software from Insitut für Nanophotonik Göttingen (Germany).

\section{Results}

After irradiation of the step edge with a single pulse with a mean fluence above $160 \mathrm{~mJ} / \mathrm{cm}^{2}$ periodic structures start to appear on the sample surface. We found LIPSS in the mean fluence range between $160 \mathrm{~mJ} / \mathrm{cm}^{2}$ and $270 \mathrm{~mJ} / \mathrm{cm}^{2}$. The mean fluence was calculated by measuring the beam energy and dividing it by the area of the laser spot on the sample. The appearing structures have a period close to the used laser wavelength of $343 \mathrm{~nm}$. For the investigated fluence region the period of the structures stays approximately constant as can be seen in Fig. 6.

The periods were measured using two different methods. The first method takes the maximum from the power spectrum of the measured AFM height profile (red points), while the error is computed by the resolution of the measurement, as well as evaluating the power spectra at different positions. The maxima in the power spectrum represent the frequencies of an existing periodicity in a given height profile and is calculated based on the 1D Fourier transformation [37]. The other method measures the mean distance between the maxima of the structures (blue points); again the error is taking into account the resolution of the measurement and also the variation of measurements at different positions of the structures. The resulting mean period using both methods is $338 \pm 10 \mathrm{~nm}$. The values of the period calculated by Eq. (3), taking the CDF from Ref. [34] and Ref. [35] are $344 \mathrm{~nm}$ and $340 \mathrm{~nm}$, respectively. 


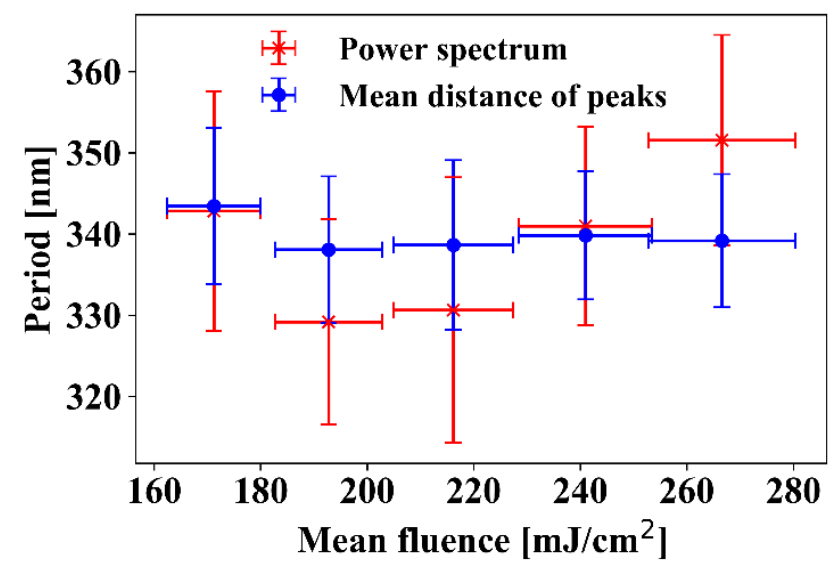

Fig. 6 Periods of the appearing LIPSS structures measured by evaluating the maximum of the power spectrum (red points) and measuring the mean distance between the maxima of the structures (blue points). The error takes into account the resolution of the AFM measurement and also variation at different positions for the same irradiation event.

We observe negligible influence of the laser fluence on the LIPSS period. However, it is known that the transient variation of the optical properties can strongly influence the absorption of laser energy and the subsequent heating dynamics [38]. Equation (3) shows that the SPP wavelength is a function of the dielectric function of the metal. As a first approximation, we used an assumption of constant dielectric function taken from Refs. [34] and [35]. It turned out that SPP periodicities with constant dielectric functions are within the experimental error bars. As a next step, we investigated how the SPP wavelength will depend on the electron temperature if we use the electron temperature-dependent dielectric function from Ref. [39]. Calculations of the SPP period using Eq. (3) with CDF at different electronic temperatures $T_{\mathrm{e}}$ as measured in Ref. [39] show a variation in the period within the precision of our measurement.

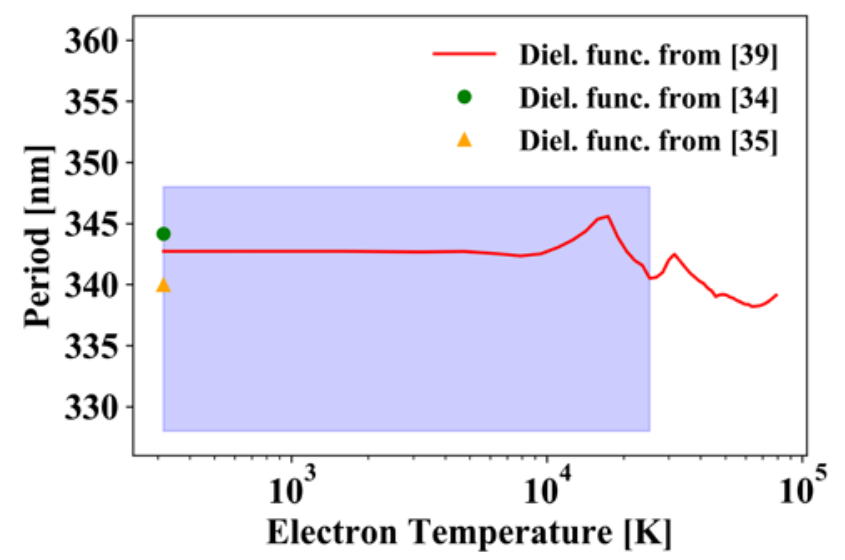

Fig. 7 Calculations of the LIPSS periods at different electronic temperatures using CDF from [39] (red line), together with calculations using constant CDF from [34] (green circle) and [35] (orange triangle). The violet shade represents the precision of our period measurement $( \pm 10 \mathrm{~nm})$ and stops at $26500 \mathrm{~K}$, the highest electronic temperature reached in the experiments, as predicted by preliminary MD-TTM simulations.
Fig. 7 shows the periods calculated for different electronic temperatures (red line), as well as the periods calculated from [34] (green circle) and [35] (orange triangle) set to room temperature together with the measurements of this work with error margin indicated by the violet shade are displayed. To estimate the maximum reached electronic temperature we have used the MD-TTM approach instead of the TTM model. It includes also phase transitions under strong non-equilibrium and generation of a variety of defects and dislocations. Preliminary MD-TTM simulations show for a fluence of $270 \mathrm{~mJ} / \mathrm{cm}^{2}$ a maximum electronic temperature of about $26500 \mathrm{~K}$. This means that in the experiments the electronic temperatures of the gold sample varied between room temperature and about $26500 \mathrm{~K}$. At this range of temperatures, the dielectric function can be approximated as constant for the given wavelength with reasonable accuracy. We plan to extend in the future our experiments to longer laser wavelengths to investigate the precision of the predictions of the SPP theory.

The LIPSS emerging after irradiation with a homogeneous beam profile decrease in height with increasing distance from the step edge. This decay in height is also predicted by the plasmonic theory. It could be reduced or completely prevented by increasing the fluence with increasing distance from the step edge. Fig. 8 shows the LIPSS with corresponding height profile for the homogeneous beam profile presented in Fig. 5 (a) and the beam profile with increasing fluence away from the step edge as shown in Fig. 5 (b) at comparable beam energies. Experiments at different positons of the scratch and with different beam energies show similar behavior as represented by the results shown in Fig. 8 .

Besides changing the decay of the structures it is also possible to change the height of the LIPSS by increasing the mean fluence of the pulse. Fig. 9 shows the maximum peakvalley modulation of the structures starting at the second peak of the structures. The first maximum is often polluted by debris from the step edge appearing after irradiation. For fluences above $230 \mathrm{~mJ} / \mathrm{cm}^{2}$ more debris, void opening [30] and partial ablation made a reliable height measurement of the undisturbed structures impossible. One sees an increase in the structure heights from about $12 \mathrm{~nm}$ to about $47 \mathrm{~nm}$. The error is calculated by measuring the height profiles at different positions on the step edge, the error of the AFM height measurement is assumed to be negligible. 

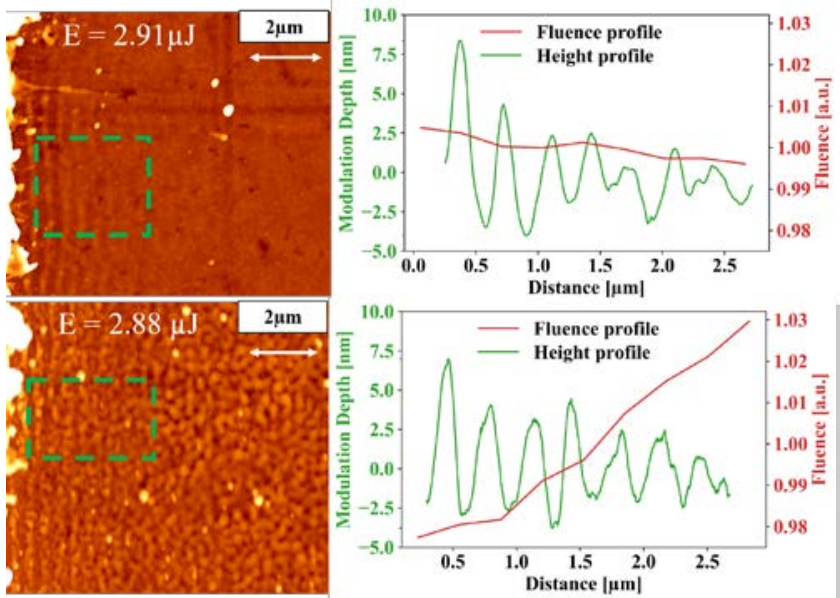

Fig. 8 On the left are the AFM images of LIPSS forming on a preexisting step edge on a gold sample after single pulse irradiation with a beam energy of $2.91 \mu \mathrm{J}$ and mean fluence of $172 \mathrm{~mJ} / \mathrm{cm}^{2}$ (top) and $2.88 \mu \mathrm{J}$ (bottom), with the beam profiles shown in Fig. 5 (a) and (b), respectively. The arrow indicates the direction of the polarization of the beam, the green box the position at which the heights were measured. On the right the corresponding AFM height measurements (green curve) are displayed. The height profiles are averaged over about 160 and 100 horizontal lines within the green boxes displayed in the left pictures for the top and bottom measurement, respectively. The red line shows the horizontal fluence profile across the beam. The maximum peak-valley modulation of the structure is in the top picture about $12 \mathrm{~nm}$, for the bottom it is about $10 \mathrm{~nm}$. In both cases the height $=0 \mathrm{~nm}$ is set to the mean height of the structure. The bottom curve shows a slower decay in height compared to the top one, while the maximum peak-valley modulation is comparable. In the lower AFM picture, as the fluence increases with increasing distance from the scratch, the surface at the right side is stronger heated, which is responsible for the increased roughness in that area.

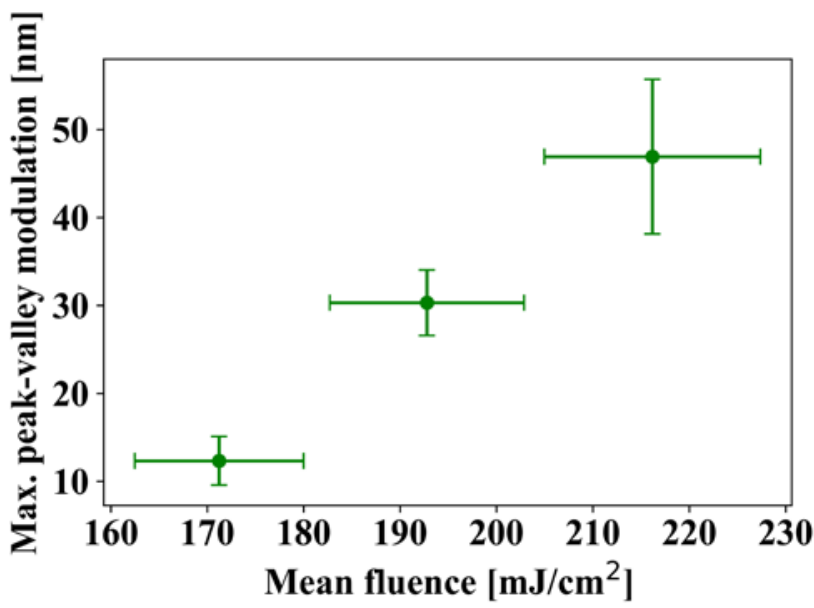

Fig. 9 The maximum peak-valley modulation of the structures starting at the second maximum shown against the used mean laser fluence. The structure height increases with increasing laser fluence. For the irradiations the homogenous beam profile visible in Fig. 5 (a) was used. The error is estimated taking measurements at different positions along the step edge, the error of the individual height measurements was assumed to be negligible.

As visible in Fig. 8, a change in the distribution of fluence within the beam profile can be used to modify the decaying behavior of the structures. In Fig. 9 it is shown that an increase in fluence leads to an overall increase in the structure height. These two changes in the beam characteristics may be used to manipulate the appearing LIPSS. From the technological point of view, the single-shot LIPSS formation method in comparison with the laser scanning technology [40] can serve as an alternative method of surface functionalization technology. However, one needs to carefully check the entire treated area for scratches and defects because they can cause inhomogeneous laser energy deposition and result in irregular LIPSS with bifurcations. From the academic point of view, the investigation of single-shot experiments with LIPSS formation is more beneficial because of a possibility of the direct comparison of theoretical predictions with experimental results on the same temporal and spatial scales and in terms of computational efficiency.

\section{Conclusion}

In this publication we show experimentally the emergence of LIPSS on a pre-structured gold sample after irradiation with a single laser pulse. The measured LIPSS periods are in good agreement with the predictions from plasmonic theory. We can answer the question of how to create LIPSS with small periodicity and homogeneous distribution of heights. First, one should ensure the SPP excitation, which we make with the help of the step edge on a gold sample. Second, one should choose a small laser wavelength to create LIPSS with small periodicity because in single-pulse experiments the LIPSS periodicity will be close to the laser wavelength. Third, one should apply laser beam shaping to achieve a more homogeneous distribution of LIPSS heights.

In the investigated fluence region the value of the fluence has negligible influence on the final periods of LIPSS, although the overall height of LIPSS is increasing with increasing the fluence of the laser beam. We show that via manipulation of the laser beam profile by adjusting a fluence slope with increasing distance from the step edge, the decay of the height of the structures may be reduced or even completely eliminated, thus facilitating the formation of LIPSS with uniform heights. As a result, the presented method of singlepulse LIPSS formation can be used in laser micro-engineering for manufacturing of surface structures with demanded properties

\section{Acknowledgments}

We acknowledge the financial support of the Deutsche Forschungsgemeinschaft, projects RE1141/14-2, IH 17/18-2, GA465/15-2.

\section{References}

[1] M. Birnbaum: J. Appl. Phys. 36, (1965) 3688.

[2] J. Bonse, S. Höhm, S. V. Kirner, A. Rosenfeld, and J. Krüger: IEEE J. Sel. Top. Quantum Electron. 23, (2017) 9000615.

[3] J. Bonse and S. Gräf: Laser Photonics Rev. 14, (2020) 2000215

[4] C. Florian, S. V. Kirner, J. Krüger, and J. Bonse: J. Laser Appl. 32, (2020) 022063.

[5] P. N. Terekhin, O. Benhayoun, S. T. Weber, D. S. Ivanov, M. E.Garcia, and B. Rethfeld: Appl. Surf. Sci. 512, (2020) 144420.

[6] J. E. Sipe, J. F. Young, J. S. Preston, and H. M. Van Driel: Phys. Rev. B 27, (1983) 1141. 
[7] J. Bonse, A. Rosenfeld, and J. Krüger: J. Appl. Phys. 106, (2009) 104910.

[8] T. Leißner, C. Lemke, J. Fiutowski, J. W. Radke, A. Klick, L. Tavares, J. Kjelstrup-Hansen, H. G. Rubahn, and M. Bauer: Phys. Rev. Lett. 111, (2013) 046802.

[9] T. Zang, H. Zang, Z. Xi, J. Du, H. Wang, Y. Lu, and P. Wang: Phys. Rev. Lett. 124, (2020) 243901.

[10] V. Bruno, C. Devault, S. Vezzoli, Z. Kudyshev, T. Huq, S. Mignuzzi, A. Jacassi, S. Saha, Y. D. Shah, S. A. Maier, D. R. Cumming, A. Boltasseva, M. Ferrera, M. Clerici, D. Faccio, R. Sapienza, and V. M. Shalaev: Phys. Rev. Lett. 124, (2020) 43902.

[11]S. A. Maier: Plasmonics: Fundamentals and Applications (Springer US, New York, NY, 2007)

[12]A. Y. Vorobyev, V. S. Makin, and C. Guo: Phys. Rev. Lett. 102, (2009) 234301.

[13] Y. Liu, Y. Brelet, Z. He, L. Yu, S. Mitryukovskiy, A. Houard, B. Forestier, A. Couairon, and A. Mysyrowicz: Phys. Rev. Lett. 110, (2013) 097601.

[14] R. D. Murphy, B. Torralva, D. P. Adams, and S. M. Yalisove: Appl. Phys. Lett. 102, (2013) 211101.

[15] R. D. Murphy, B. Torralva, D. P. Adams, and S. M. Yalisove: Appl. Phys. Lett. 104, (2014) 231117.

[16]E. L. Gurevich: Appl. Surf. Sci. 278, (2013) 52.

[17] E. L. Gurevich and S. V. Gurevich: Appl. Surf. Sci. 302, (2014) 118.

[18] S. Maragkaki, T. J. Derrien, Y. Levy, N. M. Bulgakova, A. Ostendorf, and E. L. Gurevich: Appl. Surf. Sci. 417, (2017) 88.

[19]E. L. Gurevich, Y. Levy, and N. M. Bulgakova: Nanomaterials 10, (2020) 1836.

[20] N. Ackerl and K. Wegener: J. Laser Appl. 32, (2020) 022049.

[21]S. Anisimov, B. Kapeliovich, and T. Perel'Man: Soviet J. Exp. Theor. Phys. 39, (1974) 776.

[22]B. Rethfeld, D. S. Ivanov, M. E. Garcia, and S. I. Anisimov: J. Phys. D 50, (2017) 193001.

[23] T. J. Derrien, T. Sarnet, M. Sentis, and T. Itina: J. Optoelectron. Adv. Mater. 12, (2010) 610.

[24] E. L. Gurevich, Y. Levy, S. V. Gurevich, and N. M. Bulgakova: Phys. Rev. B 95, (2017) 054305.

[25] Y. Levy, T. J.-Y. Derrien, N. M. Bulgakova, E. L. Gurevich, and T. Mocek: Appl. Surf. Sci. 374, (2016) 157.

[26] Y. Levy, N. M. Bulgakova, and T. Mocek: Proc. SPIE, Vol. 10228, (2017) 102280T.

[27] M. V. Shugaev, I. Gnilitskyi, N. M. Bulgakova, and L. V. Zhigilei: Phys. Rev. B 96, (2017) 205429.

[28]C. Y. Shih, I. Gnilitskyi, M. V. Shugaev, E. Skoulas, E. Stratakis, and L. V. Zhigilei: Nanoscale 12, (2020) 7674

[29]D. Ivanov and L. Zhigilei: Phys. Rev. B 68, (2003) 064114.

[30]D. S. Ivanov, V. P. Lipp, A. Blumenstein, F. Kleinwort, V. P.Veiko, E. Yakovlev, V. Roddatis, M. E. Garcia, B. Rethfeld, J. Ihlemann, and P. Simon: Phys. Rev. Appl., (2015) 064006.

[31]C. Lemke, T. Leißner, A. Klick, J. Fiutowski, J. W. Radke, M. Thomaschewski, J. Kjelstrup-Hansen, H. G. Rubahn, and M. Bauer: Appl. Phys. B 116, (2014) 585.

[32]A. Klick, S. de la Cruz, C. Lemke, M. Großmann, H. Beyer, J. Fiutowski, H. G. Rubahn, E. R. Méndez, and M. Bauer: Appl. Phys. B 122, (2016) 79.
[33]H. Raether: Surface Plasmons on Smooth and Rough Surfaces and on Gratings (Springer Berlin Heidelberg, Berlin, Heidelberg, 1988).

[34] R. L. Olmon, B. Slovick, T. W. Johnson, D. Shelton, S. H. Oh, G. D. Boreman, and M. B. Raschke: Phys. Rev. B 86, (2012) 235147.

[35] P. B. Johnson and R. W. Christy: Phys. Rev. B 6, (1972) 4370.

[36] P. B. Johnson and R. W. Christy: Phys. Rev. B 9, (1974) 5056.

[37] T. D. Jacobs, T. Junge and L. Pastewka: Surf. Topogr.: Metrol. Prop. 5.1 (2017) 013001.

[38] G.D. Tsibidis: J. Appl. Phys. 123, (2018) 085903.

[39] A. Blumenstein, E. S. Zijlstra, D. S. Ivanov, S. T. Weber, T. Zier, F. Kleinwort, B. Rethfeld, J. Ihlemann, P. Simon, and M. E. Garcia: Phys. Rev. B 101, (2020) 165140.

[40]I. Gnilitskyi, T.J.Y. Derrien, Y. Levy, N.M. Bulgakova, T. Mocek, L. Orazi: Sci Rep 7, (2017) 8485.

(Received: June 29, 2021, Accepted: December 29, 2021) 